\title{
Global asymptotic stability for quadratic fractional difference equation
}

Mark DiPippo, Ed J Janowski and Mustafa RS Kulenović*

${ }^{*}$ Correspondence:

kulenm@math.uri.edu

Department of Mathematics,

University of Rhode Island, Kingston,

Rhode Island 02881-0816, USA

\section{Abstract}

Consider the difference equation

$$
x_{n+1}=\frac{\alpha+\sum_{i=0}^{k} a_{i} x_{n-i}+\sum_{i=0}^{k} \sum_{j=i}^{k} a_{i j} x_{n-i} x_{n-j}}{\beta+\sum_{i=0}^{k} b_{i} x_{n-i}+\sum_{i=0}^{k} \sum_{j=i}^{k} b_{i j} x_{n-i} x_{n-j}}, \quad n=0,1, \ldots
$$

where all parameters $\alpha, \beta, a_{i}, b_{i}, a_{i j}, b_{i j}, i, j=0,1, \ldots, k$, and the initial conditions $x_{i}$ $i \in\left\{-k_{1} \ldots, 0\right\}$, are nonnegative. We investigate the asymptotic behavior of the solutions of the considered equation. We give simple explicit conditions for the global stability and global asymptotic stability of the zero or positive equilibrium of this equation.

MSC: 39A10; 39A30; 65L20

Keywords: attractivity; difference equations; rational; stability

\section{Introduction}

Consider the difference equation

$$
x_{n+1}=\frac{\alpha+\sum_{i=0}^{k} a_{i} x_{n-i}+\sum_{i=0}^{k} \sum_{j=i}^{k} a_{i j} x_{n-i} x_{n-j}}{\beta+\sum_{i=0}^{k} b_{i} x_{n-i}+\sum_{i=0}^{k} \sum_{j=i}^{k} b_{i j} x_{n-i} x_{n-j}}, \quad n=0,1, \ldots,
$$

where $k \in\{0,1, \ldots\}$, the parameters $\alpha, \beta, a_{i}, b_{i}, a_{i j}, b_{i j}, i, j=0,1, \ldots, k$, and the initial conditions $x_{i}, i \in\{-k, \ldots, 0\}$, are nonnegative and such that the denominator of Eq. (1) is always positive. The important special cases of Eq. (1) are the linear fractional equations such as the well-known Riccati equation

$$
x_{n+1}=\frac{\alpha+a_{0} x_{n}}{\beta+b_{0} x_{n}}, \quad n=0,1, \ldots
$$

the second order linear fractional difference equation

$$
x_{n+1}=\frac{\alpha+\sum_{i=0}^{1} a_{i} x_{n-i}}{\beta+\sum_{i=0}^{1} b_{i} x_{n-i}}, \quad n=0,1, \ldots
$$

and the third order linear fractional difference equation that we get from Eq. (1) for $k=2$ and $a_{i j}=b_{i j}=0$ for all $i, j$. The global behavior and the exact solutions of Eq. (2) even for real

(c) 2015 Dipippo et al. This article is distributed under the terms of the Creative Commons Attribution 4.0 International License (http://creativecommons.org/licenses/by/4.0/), which permits unrestricted use, distribution, and reproduction in any medium, provided you give appropriate credit to the original author(s) and the source, provide a link to the Creative Commons license, and indicate if changes were made. 
parameters were found in [1]. The global behavior of solutions of Eq. (3), in many subcases when one or more parameters are zero, was established in [1]. There is still one conjecture left whose answer will complete the global picture of the asymptotic behavior of solutions of Eq. (3). As far as the third order linear fractional difference equation is concerned, there is a large number of sporadic results that are systemized in a book [2]. The characterization of the global asymptotic behavior of solutions of Eq. (1) for $k=2$ seems to be much harder than for the second order Eq. (3). Consequently an attempt at giving the characterization of the global asymptotic behavior of solutions of Eq. (1) seems to be a formidable task at this time. However, by using some known global attractivity results, we can describe the global asymptotic behavior of solutions of Eq. (1) in some subspaces of the parametric space and the space of initial conditions. See [2-5] for a complete description of the behavior of some special cases of Eq. (1), in particular for the cases known as periodic trichotomies. See [6] where the difference in global behavior between the second and third order linear fractional difference equation is emphasized. The results on the global periodicity, that is, the results which describe all special cases of Eq. (1) where all solutions are periodic of the same period were obtained in $[7,8]$. Most results in $[2-5,9,10]$ are based on known global attractivity or global asymptotic stability results obtained in [1, 2, 11-14].

The special case of Eq. (1) with quadratic terms such as

$$
x_{n+1}=\frac{a x_{n}^{2}}{x_{n}+b x_{n-1}^{2}}, \quad n=0,1, \ldots
$$

where $a, b>0$ and the initial conditions $x_{-1}, x_{0} \geq 0, x_{-1}+x_{0}>0$, has only a negative equilibrium point for $a \leq 1$ and yet all solutions of Eq. (4) satisfy

$$
\lim _{n \rightarrow \infty} x_{n}=0 .
$$

A $k$ th order generalization of Eq. (4) with the same property is

$$
x_{n+1}=\frac{a x_{n}^{2}}{x_{n}+\sum_{i=1}^{k-1} b_{i} x_{n-i}^{2}}, \quad n=0,1, \ldots
$$

where $a, b_{i}>0$ and the initial conditions $x_{-k+1}, \ldots, x_{0} \geq 0, x_{-k+1}+\cdots+x_{0}>0$, when $a \leq 1$. Another special case of Eq. (1) with quadratic terms is

$$
x_{n+1}=\frac{B x_{n} x_{n-1}}{d x_{n}+e x_{n-1}}, \quad n=0,1, \ldots
$$

where $B, d, e>0$ and the initial conditions $x_{-1}, x_{0} \geq 0, x_{-1}+x_{0}>0$, it does not have an equilibrium point for $B \neq d+e$ and yet all solutions of Eq. (6) satisfy

$$
\lim _{n \rightarrow \infty} x_{n}=0, \quad \text { when } B<d+e,
$$

and

$$
\lim _{n \rightarrow \infty} x_{n}=\infty, \quad \text { when } B>d+e .
$$

Finally, when $B=d+e$, then Eq. (6) has an infinite number of the equilibrium points, each with its basin of attraction, see [15]. 
Another interesting case of Eq. (1) with quadratic terms is the equation

$$
x_{n+1}=\frac{a x_{n}^{2}}{1+x_{n}^{2}}, \quad n=0,1, \ldots
$$

where $a>0$ and $x_{0} \in \mathbb{R}$. When $a>2$ every solution of Eq. (7) converges either to the zero equilibrium or to the bigger positive equilibrium $x_{+}=\frac{a+\sqrt{a^{2}-4}}{2}$, with basins of attraction being $\mathcal{B}(0)=\left[0, x_{-}\right)$and $\mathcal{B}\left(x_{+}\right)=\left(x_{-}, \infty\right)$, where $x_{-}=\frac{a-\sqrt{a^{2}-4}}{2}$ is the smaller positive equilibrium.

None of these asymptotic behaviors which are present in the cases of Eqs. (4)-(7) are possible in the case of the linear fractional equation

$$
x_{n+1}=\frac{\alpha+\sum_{i=0}^{k} a_{i} x_{n-i}}{\beta+\sum_{i=0}^{k} b_{i} x_{n-i}}, \quad n=0,1, \ldots,
$$

and the appearance of these behaviors is caused by the presence of the quadratic terms. The results presented here have been successfully applied to some special classes of Eq. (1), see $[16,17]$ and can be applied to equations considered in [18-22].

This paper is an attempt at establishing some global stability results for the equilibrium solution(s) of Eq. (1). Our results give effective conditions for global asymptotic stability of the equilibrium solution(s) of Eq. (1) expressed in terms of the inequalities on the parameters.

\section{Preliminaries}

The following general global results will be applied to Eq. (1), see [23].

Consider the difference equation

$$
x_{n+1}=f\left(x_{n}, \ldots, x_{n-k}\right), \quad n=0,1, \ldots,
$$

where $k \in\{0,1, \ldots\}$. Sometimes it is more advantageous to investigate Eq. (8) by embedding Eq. (8) into a higher iteration of the form

$$
x_{n+l}=F\left(x_{n+l-1}, \ldots, x_{n-k}\right), \quad n=0,1, \ldots
$$

where $l \in\{1,2, \ldots\}$, see $[23,24]$ and then linearizing (rearranging) Eq. (9) so that it has the form

$$
x_{n+l}=\sum_{i=1-l}^{k} g_{i} x_{n-i}, \quad n=0,1, \ldots,
$$

where the functions $g_{i}: \mathbb{R}^{k+l} \rightarrow \mathbb{R}$.

Theorem 1 Let $l \in\{1,2, \ldots\}$ and let $a \in \mathbb{R}$. Suppose that Eq. (8) has the linearization (10) where the functions $g_{i}: \mathbb{R}^{k+l} \rightarrow \mathbb{R}$ are such that

$$
\sum_{i=1-l}^{k}\left|g_{i}\right| \leq a<1, \quad n=0,1, \ldots
$$


Then

$$
\lim _{n \rightarrow \infty} x_{n}=0
$$

As we have observed in [23], condition (11) is actually a contraction condition in the Banach contraction principle.

In addition, we will need the following stability result from [25].

Theorem 2 Suppose that Eq. (8) can be linearized into the form

$$
x_{n+1}-\bar{x}=\sum_{i=0}^{k} g_{i}\left(x_{n-i}-\bar{x}\right), \quad n=0,1, \ldots
$$

where $\bar{x}$ is an equilibrium of Eq. (8) and the functions $g_{i}: \mathbb{R}^{k+1} \rightarrow \mathbb{R}$. If $\sum_{i=0}^{k}\left|g_{i}\right| \leq 1, n \geq 0$, then the equilibrium $\bar{x}$ of Eq. (8) is stable.

The next result follows from Theorems 1 and 2.

Corollary 1 Let $a \in \mathbb{R}$. Suppose that Eq. (8) has the linearization (12), where $\bar{x}$ is a unique nonnegative equilibrium of Eq. (8) on the interval I and the functions $g_{i}: \mathbb{R}^{k+1} \rightarrow \mathbb{R}$ are such that

$$
\sum_{i=0}^{k}\left|g_{i}\right| \leq a<1, \quad n=0,1, \ldots,
$$

then the unique nonnegative equilibrium Eq. (8) is globally asymptotically stable on the interval $I$.

The next result is an analogue of the result obtained in [23].

Lemma 1 Let $m \leq \bar{x} \leq M$ and $m \leq x_{N-i} \leq M, i=0,1, \ldots, k$, for some $N \in\{0,1, \ldots\}$. Suppose that

$$
x_{n+1}-\bar{x}=\sum_{i=0}^{k} h_{i}\left(x_{n-i}-\bar{x}\right), \quad n=0,1, \ldots
$$

where the nonnegative functions $h_{i}:[0, \infty)^{k+1} \rightarrow[0, \infty)$. Assume that for this $N$

$$
\sum_{i=0}^{k} h_{i} \leq 1
$$

Then $m \leq x_{N+1} \leq M$.

\section{Main results}

In this section we investigate the stability of the unique positive equilibrium $\bar{x}$ of Eq. (1) by using Theorems 1 and 2. Observe that Eq. (1) has a zero equilibrium if and only if $\alpha=0$ 
and $\beta>0$, in which case Eq. (1) becomes

$$
x_{n+1}=\frac{\sum_{i=0}^{k} a_{i} x_{n-i}+\sum_{i=0}^{k} \sum_{j=i}^{k} a_{i j} x_{n-i} x_{n-j}}{\beta+\sum_{i=0}^{k} b_{i} x_{n-i}+\sum_{i=0}^{k} \sum_{j=i}^{k} b_{i j} x_{n-i} x_{n-j}}, \quad n=0,1, \ldots
$$

Equation (13) yields the nonzero equilibrium points

$$
\bar{x}=\frac{-\left(\sum_{i=0}^{k} b_{i}-\sum_{i=0}^{k} \sum_{j=i}^{k} a_{i j}\right) \pm \sqrt{\left(\sum_{i=0}^{k} b_{i}-\sum_{i=0}^{k} \sum_{j=i}^{k} a_{i j}\right)^{2}-4\left(\beta-\sum_{i=0}^{k} a_{i}\right) \sum_{i=0}^{k} \sum_{j=i}^{k} b_{i j}}}{2 \sum_{i=0}^{k} \sum_{j=i}^{k} b_{i j}} .
$$

Thus if $\beta \geq \sum_{i=0}^{k} a_{i}$ and $\sum_{i=0}^{k} b_{i}>\sum_{i=0}^{k} \sum_{j=i}^{k} a_{i j}$, then there is no positive equilibrium. The following result shows that there is an interval in which every solution of Eq. (13) converges to the zero equilibrium. For convenience of notation, let $Q$ denote the denominator of Eq. (1), that is,

$$
Q=\beta+\sum_{i=0}^{k} b_{i} x_{n-i}+\sum_{i=0}^{k} \sum_{j=i}^{k} b_{i j} x_{n-i} x_{n-j}
$$

Theorem 3 Let $M \in(0, \infty)$ be such that $M<\frac{\beta-\sum_{i=0}^{k} a_{i}}{\sum_{i=0}^{k} \sum_{j=i}^{k} a_{i j}}$. Assume that there is no positive equilibrium. Then the zero equilibrium of Eq. (13) is globally asymptotically stable on the interval $[0, M)$.

Proof Observe that Eq. (13) can be linearized into the form (10) where $l=1$ as follows for $n \geq 0$

$$
x_{n+1}=\frac{a_{0}+\sum_{j=0}^{k} a_{0 j} x_{n-j}}{Q} x_{n}+\frac{a_{1}+\sum_{j=1}^{k} a_{1 j} x_{n-j}}{Q} x_{n-1}+\cdots+\frac{a_{k}+a_{k k} x_{n-k}}{Q} x_{n-k} .
$$

Then, for $i=0, \ldots, k$,

$$
\left|g_{i}\right|=\frac{a_{i}+\sum_{j=i}^{k} a_{i j} x_{n-j}}{Q}, \quad n=0,1, \ldots
$$

Let $\left\{x_{n}\right\}$ be a solution of Eq. (13) where $\max \left\{x_{0}, \ldots, x_{-k}\right\} \leq M$. Then for $n=0$

$$
\sum_{i=0}^{k}\left|g_{i}\right|=\frac{\sum_{i=0}^{k} a_{i}+\sum_{i=0}^{k} \sum_{j=i}^{k} a_{i j} x_{-j}}{\beta+\sum_{i=0}^{k} b_{i} x_{-i}+\sum_{i=0}^{k} \sum_{j=i}^{k} b_{i j} x_{-i} x_{-j}} \leq \frac{\sum_{i=0}^{k} a_{i}+M \sum_{i=0}^{k} \sum_{j=i}^{k} a_{i j}}{\beta}<1 .
$$

By Lemma 1 with $\bar{x}=0, h_{i}=\left|g_{i}\right|, i=0, \ldots, k$, and $N=0$, we get that $x_{1} \leq M$. Hence $x_{i} \leq M$ for $i=1,0, \ldots,-k$. Then for $n=1$

$$
\sum_{i=0}^{k}\left|g_{i}\right|=\frac{\sum_{i=0}^{k} a_{i}+\sum_{i=0}^{k} \sum_{j=i}^{k} a_{i j} x_{1-j}}{\beta+\sum_{i=0}^{k} b_{i} x_{1-i}+\sum_{i=0}^{k} \sum_{j=i}^{k} b_{i j} x_{1-i} x_{1-j}} \leq \frac{\sum_{i=0}^{k} a_{i}+M \sum_{i=0}^{k} \sum_{j=i}^{k} a_{i j}}{\beta}<1 .
$$


Again using Lemma 1 with $\bar{x}=0, h_{i}=\left|g_{i}\right|, i=0, \ldots, k$, and $N=1$, we get that $x_{2} \leq M$. Hence $x_{i} \leq M$ for $i=2,1, \ldots,-k$. Then for $n=2$

$$
\sum_{i=0}^{k}\left|g_{i}\right|=\frac{\sum_{i=0}^{k} a_{i}+\sum_{i=0}^{k} \sum_{j=i}^{k} a_{i j} x_{2-j}}{\beta+\sum_{i=0}^{k} b_{i} x_{2-i}+\sum_{i=0}^{k} \sum_{j=i}^{k} b_{i j} x_{2-i} x_{2-j}} \leq \frac{\sum_{i=0}^{k} a_{i}+M \sum_{i=0}^{k} \sum_{j=i}^{k} a_{i j}}{\beta}<1 .
$$

By induction we get that for $n \geq 0$

$$
\sum_{i=0}^{k}\left|g_{i}\right|=\frac{\sum_{i=0}^{k} a_{i}+\sum_{i=0}^{k} \sum_{j=i}^{k} a_{i j} x_{n-j}}{\beta+\sum_{i=0}^{k} b_{i} x_{n-i}+\sum_{i=0}^{k} \sum_{j=i}^{k} b_{i j} x_{n-i} x_{n-j}} \leq \frac{\sum_{i=0}^{k} a_{i}+M \sum_{i=0}^{k} \sum_{j=i}^{k} a_{i j}}{\beta}<1,
$$

and so the result follows from Corollary 1 where $\bar{x}=0$ and the interval is $[0, M)$.

All equilibrium solutions of Eq. (1) satisfy the equilibrium equation

$$
\sum_{i=0}^{k} \sum_{j=i}^{k} b_{i j} x^{3}+\left(\sum_{i=0}^{k} b_{i}-\sum_{i=0}^{k} \sum_{j=i}^{k} a_{i j}\right) x^{2}+\left(\beta-\sum_{i=0}^{k} a_{i}\right) x-\alpha=0,
$$

which can be rewritten as

$$
\alpha-\beta x+x \sum_{i=0}^{k}\left(a_{i}-x b_{i}\right)+x^{2} \sum_{i=0}^{k} \sum_{j=i}^{k}\left(a_{i j}-x b_{i j}\right)=0 .
$$

Equilibrium Eq. (14) has at least one nonnegative zero and it may have between 0 and 3 positive zeros. When $\alpha>0$ and either $\sum_{i=0}^{k} b_{i}-\sum_{i=0}^{k} \sum_{j=i}^{k} a_{i j} \geq 0, \beta-\sum_{i=0}^{k} a_{i} \geq 0$ or $\sum_{i=0}^{k} b_{i}-\sum_{i=0}^{k} \sum_{j=i}^{k} a_{i j} \geq 0, \beta-\sum_{i=0}^{k} a_{i} \leq 0$ or $\sum_{i=0}^{k} b_{i}-\sum_{i=0}^{k} \sum_{j=i}^{k} a_{i j} \leq 0, \beta-\sum_{i=0}^{k} a_{i} \leq 0$, Descartes rule of sign implies that there is a unique positive equilibrium of Eq. (1).

If $\bar{x}>0$ is an equilibrium, then for $n \geq 0$

$$
\begin{aligned}
x_{n+1}-\bar{x} & =\frac{\alpha+\sum_{i=0}^{k} a_{i} x_{n-i}+\sum_{i=0}^{k} \sum_{j=i}^{k} a_{i j} x_{n-i} x_{n-j}}{\beta+\sum_{i=0}^{k} b_{i} x_{n-i}+\sum_{i=0}^{k} \sum_{j=i}^{k} b_{i j} x_{n-i} x_{n-j}}-\bar{x} \\
& =\frac{\alpha-\beta \bar{x}+\sum_{i=0}^{k}\left(a_{i}-b_{i} \bar{x}\right) x_{n-i}+\sum_{i=0}^{k} \sum_{j=i}^{k}\left(a_{i j}-b_{i j} \bar{x}\right) x_{n-i} x_{n-j}}{Q}, \\
& =\frac{\sum_{i=0}^{k}\left(a_{i}-b_{i} \bar{x}\right)\left(x_{n-i}-\bar{x}\right)+\sum_{i=0}^{k} \sum_{j=i}^{k}\left(a_{i j}-b_{i j} \bar{x}\right)\left(x_{n-i} x_{n-j}-\bar{x}^{2}\right)+R}{Q},
\end{aligned}
$$

where in view of Eqs. (14), (15)

$$
R=\alpha-\beta \bar{x}+\bar{x} \sum_{i=0}^{k}\left(a_{i}-b_{i} \bar{x}\right)+\bar{x}^{2} \sum_{i=0}^{k} \sum_{j=i}^{k}\left(a_{i j}-b_{i j} \bar{x}\right)=0 .
$$

Now applying the identity

$$
x_{n-i} x_{n-j}-\bar{x}^{2}=x_{n-i}\left(x_{n-j}-\bar{x}\right)+\bar{x}\left(x_{n-i}-\bar{x}\right), \quad i, j=0,1, \ldots
$$


we get that for $n \geq 0$

$$
x_{n+1}-\bar{x}=\frac{\sum_{i=0}^{k}\left(a_{i}-b_{i} \bar{x}\right)\left(x_{n-i}-\bar{x}\right)+\sum_{i=0}^{k} \sum_{j=i}^{k}\left(a_{i j}-b_{i j} \bar{x}\right) \bar{x}\left(x_{n-i}-\bar{x}\right)+\sum_{i=0}^{k} \sum_{j=i}^{k}\left(a_{i j}-b_{i j} \bar{x}\right) x_{n-i}\left(x_{n-j}-\bar{x}\right)}{Q} .
$$

Observe that for $n \geq 0$

$$
\sum_{i=0}^{k} \sum_{j=i}^{k}\left(a_{i j}-b_{i j} \bar{x}\right) x_{n-i}\left(x_{n-j}-\bar{x}\right)=\sum_{i=0}^{k} \sum_{j=0}^{i}\left(a_{j i}-b_{j i} \bar{x}\right) x_{n-j}\left(x_{n-i}-\bar{x}\right) .
$$

Thus for $n \geq 0$

$$
\begin{aligned}
x_{n+1}-\bar{x} & =\frac{\sum_{i=0}^{k}\left(a_{i}-b_{i} \bar{x}\right)\left(x_{n-i}-\bar{x}\right)+\sum_{i=0}^{k} \sum_{j=i}^{k}\left(a_{i j}-b_{i j} \bar{x}\right) \bar{x}\left(x_{n-i}-\bar{x}\right)+\sum_{i=0}^{k} \sum_{j=0}^{i}\left(a_{j i}-b_{j i} \bar{x}\right) x_{n-j}\left(x_{n-i}-\bar{x}\right)}{Q} \\
& =\frac{\sum_{i=0}^{k}\left(a_{i}-b_{i} \bar{x}\right)\left(x_{n-i}-\bar{x}\right)+\sum_{i=0}^{k}\left(\sum_{j=i}^{k}\left(a_{i j}-b_{i j} \bar{x}\right) \bar{x}+\sum_{j=0}^{i}\left(a_{j i}-b_{j i} \bar{x}\right) x_{n-j}\right)\left(x_{n-i}-\bar{x}\right)}{Q} .
\end{aligned}
$$

Therefore for $n \geq 0$

$$
x_{n+1}-\bar{x}=\sum_{i=0}^{k} \frac{\left(a_{i}-b_{i} \bar{x}\right)+\sum_{j=i}^{k}\left(a_{i j}-b_{i j} \bar{x}\right) \bar{x}+\sum_{j=0}^{i}\left(a_{j i}-b_{j i} \bar{x}\right) x_{n-j}}{\beta+\sum_{i=0}^{k} b_{i} x_{n-i}+\sum_{i=0}^{k} \sum_{j=i}^{k} b_{i j} x_{n-i} x_{n-j}}\left(x_{n-i}-\bar{x}\right) .
$$

Equation (16) is the linearized equation of Eq. (1) of the form (12) where for $i=0, \ldots, k$

$$
g_{i}=\frac{\left(a_{i}-b_{i} \bar{x}\right)+\sum_{j=i}^{k}\left(a_{i j}-b_{i j} \bar{x}\right) \bar{x}+\sum_{j=0}^{i}\left(a_{j i}-b_{j i} \bar{x}\right) x_{n-j}}{\beta+\sum_{i=0}^{k} b_{i} x_{n-i}+\sum_{i=0}^{k} \sum_{j=i}^{k} b_{i j} x_{n-i} x_{n-j}}, \quad n=0,1, \ldots
$$

We can now obtain easy-to-check conditions which show when the positive equilibrium of Eq. (1) is globally asymptotically stable. We will then apply these conditions to various cases of Eq. (1).

Theorem 4 Assume that Eq. (1) has a unique positive equilibrium $\bar{x}$ and there exist $L \geq 0$ and $U, N>0$ such that for every solution $\left\{x_{n}\right\}$ of Eq. (1) $L \leq x_{n} \leq U$ for all $n \geq N$ and

$$
\sum_{i=0}^{k}\left|a_{i}-b_{i} \bar{x}\right|+(U+\bar{x}) \sum_{i=0}^{k} \sum_{j=i}^{k}\left|a_{i j}-b_{i j} \bar{x}\right|<\beta+L \sum_{i=0}^{k} b_{i}+L^{2} \sum_{i=0}^{k} \sum_{j=i}^{k} b_{i j}
$$

where $\beta+L>0$. Then the unique positive equilibrium $\bar{x}$ of Eq. (1) is globally asymptotically stable on the interval $[0, \infty)$.

Proof As we have seen Eq. (1) can be written in the form of the linearized Eq. (16), where the coefficients $g_{i}$ are given as (17).

We have for $n \geq 0$

$$
Q=\beta+\sum_{i=0}^{k} b_{i} x_{n-i}+\sum_{i=0}^{k} \sum_{j=i}^{k} b_{i j} x_{n-i} x_{n-j} \geq \beta+L \sum_{i=0}^{k} b_{i}+L^{2} \sum_{i=0}^{k} \sum_{j=i}^{k} b_{i j}
$$


and so for $i=0, \ldots, k$ and $n \geq 0$

$$
\left|g_{i}\right| \leq \frac{\left|a_{i}-b_{i} \bar{x}\right|+\sum_{j=i}^{k}\left|a_{i j}-b_{i j} \bar{x}\right| \bar{x}+\sum_{j=0}^{i}\left|a_{j i}-b_{j i} \bar{x}\right| x_{n-j}}{Q} .
$$

Thus for $n \geq 0$

$$
\sum_{i=0}^{k}\left|g_{i}\right| \leq \frac{\sum_{i=0}^{k}\left|a_{i}-b_{i} \bar{x}\right|+\sum_{i=0}^{k} \sum_{j=i}^{k}\left|a_{i j}-b_{i j} \bar{x}\right| \bar{x}+\sum_{i=0}^{k} \sum_{j=0}^{i}\left|a_{j i}-b_{j i} \bar{x}\right| x_{n-j}}{Q}
$$

By rearranging the terms we can show that for $n \geq 0$

$$
\sum_{i=0}^{k} \sum_{j=0}^{i}\left|a_{j i}-b_{j i} \bar{x}\right| x_{n-j}=\sum_{i=0}^{k} \sum_{j=i}^{k}\left|a_{i j}-b_{i j} \bar{x}\right| x_{n-i} .
$$

Thus for $n \geq 0$

$$
\sum_{i=0}^{k}\left|g_{i}\right| \leq \frac{\sum_{i=0}^{k}\left|a_{i}-b_{i} \bar{x}\right|+\sum_{i=0}^{k} \sum_{j=i}^{k}\left|a_{i j}-b_{i j} \bar{x}\right| \bar{x}+\sum_{i=0}^{k} \sum_{j=i}^{k}\left|a_{i j}-b_{i j} \bar{x}\right| x_{n-i}}{Q},
$$

and so

$$
\sum_{i=0}^{k}\left|g_{i}\right| \leq \frac{\sum_{i=0}^{k}\left|a_{i}-b_{i} \bar{x}\right|+\sum_{i=0}^{k} \sum_{j=i}^{k}\left|a_{i j}-b_{i j} \bar{x}\right|\left(\bar{x}+x_{n-i}\right)}{Q} .
$$

In view of (18) and (19), we obtain

$$
\sum_{i=0}^{k}\left|g_{i}\right| \leq \frac{\sum_{i=0}^{k}\left|a_{i}-b_{i} \bar{x}\right|+\sum_{i=0}^{k} \sum_{j=i}^{k}\left|a_{i j}-b_{i j} \bar{x}\right|(\bar{x}+U)}{\beta+L \sum_{i=0}^{k} b_{i}+L^{2} \sum_{i=0}^{k} \sum_{j=i}^{k} b_{i j}}<1
$$

for $n \geq 0$. So by Corollary $1 \bar{x}$ is globally asymptotically stable on the interval $[L, U]$. By assumption every solution of Eq. (1) enters the interval $[L, U]$ and so the result follows.

From (18) we see that establishing a lower bound for all the solutions of Eq. (1) will give us a better result. We present some of these cases.

Remark 1 The results on boundedness of all solutions of Eq. (1) are well known, see [2, 24]. For instance, if for every $i, j \in\{0, \ldots, k\}$ such that $b_{i}>0, b_{i j}>0$ we have $a_{i}>0, a_{i j}>0$, then the uniform lower bound $L$ for all solutions $\left\{x_{n}\right\}$ of Eq. (1) for $n \geq 1$ is

$$
L=\frac{\min \left\{\alpha, a_{i}, a_{i j} \mid a_{i}>0, a_{i j}>0\right\}}{\max \left\{\beta, b_{i}, b_{i j}>0 \mid b_{i}>0, b_{i j}>0\right\}} .
$$

On the other hand, if for every $i, j \in\{0, \ldots, k\}$ such that $a_{i}, a_{i j}>0$ we have $b_{i}, b_{i j}>0$, then the uniform lower bound $L$ for all solutions of Eq. (1) for $n \geq 1$ is

$$
L=\frac{\min \left\{\alpha, a_{i}, a_{i j} \mid a_{i}, a_{i j}>0\right\}}{\max \left\{U \sum_{j, a_{j}=0} b_{j}, U \sum_{i, j, a_{i j}=0} b_{i j}, \beta, b_{i}, b_{i j} \mid b_{i}>0\right\}},
$$


where

$$
U=\frac{\max \left\{\alpha, a_{i}, a_{i j} \mid a_{i}, a_{i j}>0\right\}}{\min \left\{\beta, b_{i}, b_{i j} \mid b_{i}, b_{i j}>0\right\}} .
$$

The next result follows from Lemma 1 and can be used to find the part of the basin of attraction of a positive equilibrium in the case when there are several positive equilibrium points. The proof of this result is similar to the proof of Theorem 3 and it will be omitted.

Theorem 5 Let $M=\max \left\{\bar{x}, x_{-i}: i=0, \ldots, k\right\}$ be such that

$$
\sum_{i=0}^{k}\left|a_{i}-b_{i} \bar{x}\right|+(M+\bar{x}) \sum_{i=0}^{k} \sum_{j=i}^{k}\left|a_{i j}-b_{i j} \bar{x}\right|<\beta+m \sum_{i=0}^{k} b_{i}+m^{2} \sum_{i=0}^{k} \sum_{j=i}^{k} b_{i j}
$$

where $0 \leq m=\min \left\{\bar{x}, x_{-i}: i=0, \ldots, k\right\}$ is such that $\beta+m>0$. Then the equilibrium $\bar{x}$ of Eq. (1) is globally asymptotically stable on the interval $[m, M]$.

The following result is a consequence of Theorem 4 in some special cases when the unique positive equilibrium satisfies some specific conditions.

Theorem 6 Assume that $\beta>0$ and $\bar{x}$ is the unique positive equilibrium of Eq. (1). Suppose there exist $L \geq 0$ and $U, N>0$ such that for every solution $\left\{x_{n}\right\}$ of $E q$. (1) $L \leq x_{n} \leq U$ for all $n \geq N$. Then the positive equilibrium $\bar{x}$ of Eq. (1) is globally asymptotically stable on the interval $[0, \infty)$ provided one of the following holds:

(1) $a_{i}=\bar{x} b_{i}, a_{i j}=\bar{x} b_{i j}$ for all $i, j \in\{0, \ldots, k\}$;

(2) $a_{i} \geq \bar{x} b_{i}, a_{i j} \geq \bar{x} b_{i j}$ for all $i, j \in\{0, \ldots, k\}$ and $\alpha \geq 0$ and

$$
U \sum_{i=0}^{k} \sum_{j=i}^{k}\left(a_{i j}-\bar{x} b_{i j}\right)<\frac{\alpha}{\bar{x}}+L \sum_{i=0}^{k} b_{i}+L^{2} \sum_{i=0}^{k} \sum_{j=i}^{k} b_{i j}
$$

(3) $a_{i} \leq \bar{x} b_{i}, a_{i j} \leq \bar{x} b_{i j}$ for all $i, j \in\{0, \ldots, k\}$ and

$$
U \sum_{i=0}^{k} \sum_{j=i}^{k}\left(\bar{x} b_{i j}-a_{i j}\right)<2 \beta-\frac{\alpha}{\bar{x}}+L \sum_{i=0}^{k} b_{i}+L^{2} \sum_{i=0}^{k} \sum_{j=i}^{k} b_{i j} .
$$

Proof The positive equilibrium $\bar{x}$ of Eq. (1) satisfies

$$
\beta-\frac{\alpha}{\bar{x}}=\sum_{i=0}^{k} a_{i}-\bar{x}^{2} \sum_{i=0}^{k} \sum_{j=i}^{k} b_{i j}-\bar{x}\left(\sum_{i=0}^{k} b_{i}-\sum_{i=0}^{k} \sum_{j=i}^{k} a_{i j}\right) .
$$

(1) Let $a_{i}=\bar{x} b_{i}, a_{i j}=\bar{x} b_{i j}$ for all $i, j \in\{0, \ldots, k\}$. Then $\sum_{i=0}^{k} a_{i}=\bar{x} \sum_{i=0}^{k} b_{i}$ and $\sum_{i=0}^{k} \sum_{j=i}^{k} a_{i j}=\bar{x} \sum_{i=0}^{k} \sum_{j=i}^{k} b_{i j}$, which by (23) implies $\beta=\frac{\alpha}{\bar{x}}$. Then Eq. (1) becomes for $n \geq 0$

$$
\begin{aligned}
x_{n+1} & =\frac{\alpha+\sum_{i=0}^{k} a_{i} x_{n-i}+\sum_{i=0}^{k} \sum_{j=i}^{k} a_{i j} x_{n-i} x_{n-j}}{\beta+\sum_{i=0}^{k} b_{i} x_{n-i}+\sum_{i=0}^{k} \sum_{j=i}^{k} b_{i j} x_{n-i} x_{n-j}} \\
& =\frac{\beta \bar{x}+\bar{x} \sum_{i=0}^{k} b_{i} x_{n-i}+\bar{x} \sum_{i=0}^{k} \sum_{j=i}^{k} b_{i j} x_{n-i} x_{n-j}}{\beta+\sum_{i=0}^{k} b_{i} x_{n-i}+\sum_{i=0}^{k} \sum_{j=i}^{k} b_{i j} x_{n-i} x_{n-j}}=\bar{x} .
\end{aligned}
$$


(2) In view of our assumption for $i, j \in\{0, \ldots, k\}$, we have $\left|a_{i}-\bar{x} b_{i}\right|=a_{i}-\bar{x} b_{i},\left|a_{i j}-\bar{x} b_{i j}\right|=$ $a_{i j}-\bar{x} b_{i j}$. By using (23) we obtain

$$
\begin{aligned}
\sum_{i=0}^{k}\left|a_{i}-\bar{x} b_{i}\right|+(U+\bar{x}) \sum_{i=0}^{k} \sum_{j=i}^{k}\left|a_{i j}-\bar{x} b_{i j}\right| & =\sum_{i=0}^{k} a_{i}-\bar{x} \sum_{i=0}^{k} b_{i}+(U+\bar{x}) \sum_{i=0}^{k} \sum_{j=i}^{k}\left(a_{i j}-\bar{x} b_{i j}\right) \\
& =\beta-\frac{\alpha}{\bar{x}}+U \sum_{i=0}^{k} \sum_{j=i}^{k}\left(a_{i j}-\bar{x} b_{i j}\right) .
\end{aligned}
$$

Now condition (18) is simplified to

$$
U \sum_{i=0}^{k} \sum_{j=i}^{k}\left(a_{i j}-\bar{x} b_{i j}\right)<\frac{\alpha}{\bar{x}}+L \sum_{i=0}^{k} b_{i}+L^{2} \sum_{i=0}^{k} \sum_{j=i}^{k} b_{i j}
$$

and the result follows from Theorem 4.

(3) In this case we have

$$
\begin{aligned}
\sum_{i=0}^{k}\left|a_{i}-\bar{x} b_{i}\right|+(U+\bar{x}) \sum_{i=0}^{k} \sum_{j=i}^{k}\left|a_{i j}-\bar{x} b_{i j}\right| & =\bar{x} \sum_{i=0}^{k} b_{i}-\sum_{i=0}^{k} a_{i}+(U+\bar{x}) \sum_{i=0}^{k} \sum_{j=i}^{k}\left(\bar{x} b_{i j}-a_{i j}\right) \\
& =\frac{\alpha}{\bar{x}}-\beta+U \sum_{i=0}^{k} \sum_{j=i}^{k}\left(\bar{x} b_{i j}-a_{i j}\right) .
\end{aligned}
$$

In view of our assumption,

$$
\frac{\alpha}{\bar{x}}-\beta+U \sum_{i=0}^{k} \sum_{j=i}^{k}\left(\bar{x} b_{i j}-a_{i j}\right)<\beta+L \sum_{i=0}^{k} b_{i}+L^{2} \sum_{i=0}^{k} \sum_{j=i}^{k} b_{i j}
$$

and so the result follows from Theorem 4.

Many cases of Eq. (1) have some combination of $a_{i}<\bar{x} b_{i}, a_{i}>\bar{x} b_{i}$ and $a_{i}=\bar{x} b_{i}$. In view of this we will adopt the following notations, where $I_{>}=\left\{i \mid\right.$ such that $\left.a_{i}>\bar{x} b_{i}\right\}$, $I_{=}=\left\{i \mid\right.$ such that $\left.a_{i}=\bar{x} b_{i}\right\}, I_{<}=\left\{i \mid\right.$ such that $\left.a_{i}<\bar{x} b_{i}\right\}$ :

$$
\begin{aligned}
& \left\{\begin{array}{l}
A_{S}=\sum_{i \in I_{>}} a_{i}=\text { the sum of all the } a_{i}{ }^{\prime} \mathrm{s} \\
B_{S}=\sum_{i \in I_{>}} b_{i}=\text { the sum of all the } b_{i}{ }^{\prime} \mathrm{s}
\end{array}\right\} \text { such that } a_{i}>\bar{x} b_{i}, \\
& \left\{\begin{array}{l}
A_{N}=\sum_{i \in I_{=}} a_{i}=\text { the sum of all the } a_{i}^{\prime} \text { 's } \\
B_{N}=\sum_{i \in I_{=}} b_{i}=\text { the sum of all the } b_{i} \text { 's }
\end{array}\right\} \text { such that } a_{i}=\bar{x} b_{i}, \\
& \left\{\begin{array}{l}
A_{R}=\sum_{i \in I_{<}} a_{i}=\text { the sum of all the } a_{i}^{\prime} \mathrm{s} \\
B_{R}=\sum_{i \in I_{<}} b_{i}=\text { the sum of all the } b_{i} \text { 's }
\end{array}\right\} \text { such that } a_{i}<\bar{x} b_{i} .
\end{aligned}
$$

Then $A_{S}+A_{N}+A_{R}=\sum_{i=0}^{k} a_{i}$ and $B_{S}+B_{N}+B_{R}=\sum_{i=0}^{k} b_{i}$. Also $A_{S}>\bar{x} B_{S}, A_{N}=\bar{x} B_{N}$ and $A_{R}<\bar{x} B_{R}$.

Similarly define

$$
\left\{\begin{array}{l}
\bar{A}_{S}=\sum_{i=0}^{k} \sum_{j=i}^{k} a_{i j}=\text { the sum of all the } a_{i j}{ }^{\prime} \mathrm{s} \\
\bar{B}_{S}=\sum_{i=0}^{k} \sum_{j=i}^{k} b_{i j}=\text { the sum of all the } b_{i j} \text { 's }
\end{array}\right\} \text { such that } a_{i j}>\bar{x} b_{i j}
$$




$$
\begin{aligned}
& \left\{\begin{array}{l}
\bar{A}_{N}=\sum_{i=0}^{k} \sum_{j=i}^{k} a_{i j}=\text { the sum of all the } a_{i j} \text { 's } \\
\bar{B}_{N}=\sum_{i=0}^{k} \sum_{j=i}^{k} b_{i j}=\text { the sum of all the } b_{i j} \text { 's }
\end{array}\right\} \text { such that } a_{i j}=\bar{x} b_{i j}, \\
& \left\{\begin{array}{l}
\bar{A}_{R}=\sum_{i=0}^{k} \sum_{j=i}^{k} a_{i j}=\text { the sum of all the } a_{i j} \text { 's } \\
\bar{B}_{R}=\sum_{i=0}^{k} \sum_{j=i}^{k} b_{i j}=\text { the sum of all the } b_{i j} \text { 's }
\end{array}\right\} \text { such that } a_{i j}<\bar{x} b_{i j} .
\end{aligned}
$$

Then $\bar{A}_{S}+\bar{A}_{N}+\bar{A}_{R}=\sum_{i=0}^{k} \sum_{j=i}^{k} a_{i j}$ and $\bar{B}_{S}+\bar{B}_{N}+\bar{B}_{R}=\sum_{i=0}^{k} \sum_{j=i}^{k} b_{i j}$. Also $\bar{A}_{S}>\bar{x} \bar{B}_{S}, \bar{A}_{N}=$ $\bar{x} \bar{B}_{N}, \bar{A}_{R}<\bar{x} \bar{B}_{R}$ and

$$
\begin{aligned}
\sum_{i=0}^{k} \sum_{j=i}^{k}\left|a_{i j}-\bar{x} b_{i j}\right| & =\left(\bar{A}_{S}-\bar{x} \bar{B}_{S}\right)+\left(\bar{x} \bar{B}_{N}-\bar{A}_{N}\right)+\left(\bar{x} \bar{B}_{R}-\bar{A}_{R}\right) \\
& =\sum_{i=0}^{k} \sum_{j=i}^{k} \bar{x} b_{i j}-\sum_{i=0}^{k} \sum_{j=i}^{k} a_{i j}+2\left(\bar{A}_{S}-\bar{x} \bar{B}_{S}\right) .
\end{aligned}
$$

Corollary 2 Suppose that the assumptions of Theorem 6 are satisfied. Let $i, j \in\{0, \ldots, k\}$. Assume that

(a) For some $i$,j's $a_{i j}>\bar{x} b_{i j}$ and for other $i, j$ 's $a_{i j}<\bar{x} b_{i j}$.

(b) For some i's $a_{i}>\bar{x} b_{i}$ and for other i's $a_{i}<\bar{x} b_{i}$.

(c)

$$
\begin{aligned}
\frac{\alpha}{\bar{x}}+2\left(A_{S}-\bar{x} B_{S}\right)+(U+2 \bar{x})\left(\bar{A}_{S}-\bar{x} \bar{B}_{S}\right)+U\left(\bar{x} B_{R}-\bar{A}_{R}\right) \\
\quad<2 \beta+L \sum_{i=0}^{k} b_{i}+L^{2} \sum_{i=0}^{k} \sum_{j=i}^{k} b_{i j} .
\end{aligned}
$$

Then the positive equilibrium $\bar{x}$ of Eq. (1) is globally asymptotically stable on the interval $[0, \infty)$.

Proof In view of the equilibrium Eq. (15) and by assumption (c), we have that

$$
\begin{aligned}
& \sum_{i=0}^{k}\left|a_{i}-\bar{x} b_{i}\right|+(U+\bar{x}) \sum_{i=0}^{k} \sum_{j=i}^{k}\left|a_{i j}-\bar{x} b_{i j}\right| \\
& =\bar{x} \sum_{i=0}^{k} b_{i}-\sum_{i=0}^{k} a_{i}+2\left(A_{S}-\bar{x} B_{S}\right)+2(U+\bar{x})\left(\bar{A}_{S}-\bar{x} \bar{B}_{S}\right)+(U+\bar{x}) \sum_{i=0}^{k} \sum_{j=i}^{k} \bar{x} b_{i j} \\
& \quad-(U+\bar{x}) \sum_{i=0}^{k} \sum_{j=i}^{k} a_{i j} \\
& =\sum_{i=0}^{k}\left(\bar{x} b_{i}-a_{i}\right)+\bar{x} \sum_{i=0}^{k} \sum_{j=i}^{k}\left(\bar{x} b_{i j}-a_{i j}\right)+2\left(A_{S}-\bar{x} B_{S}\right)+(2 U+2 \bar{x})\left(\bar{A}_{S}-\bar{x} \bar{B}_{S}\right) \\
& \quad+U \sum_{i=0}^{k} \sum_{j=i}^{k}\left(\bar{x} b_{i j}-a_{i j}\right) \\
& =\frac{\alpha}{\bar{x}}-\beta+2\left(A_{S}-\bar{x} B_{S}\right)+(2 U+2 \bar{x})\left(\bar{A}_{S}-\bar{x} \bar{B}_{S}\right)+U \bar{x}\left(\bar{B}_{S}+\bar{B}_{N}+\bar{B}_{R}\right) \\
& \quad-U\left(\bar{A}_{S}+\bar{A}_{N}+\bar{A}_{R}\right)
\end{aligned}
$$




$$
\begin{aligned}
& =\frac{\alpha}{\bar{x}}-\beta+2\left(A_{S}-\bar{x} B_{S}\right)+(U+2 \bar{x})\left(\bar{A}_{S}-\bar{x} \bar{B}_{S}\right)+U\left(\bar{x} \bar{B}_{R}-\bar{A}_{R}\right) \\
& <\beta+L \sum_{i=0}^{k} b_{i}+L^{2} \sum_{i=0}^{k} \sum_{j=i}^{k} b_{i j} .
\end{aligned}
$$

Now, the conclusion follows from Theorem 4.

In the case of general second order quadratic fractional equation of the form

$$
x_{n+1}=\frac{A x_{n}^{2}+B x_{n} x_{n-1}+C x_{n-1}^{2}+D x_{n}+E x_{n-1}+F}{a x_{n}^{2}+b x_{n} x_{n-1}+c x_{n-1}^{2}+d x_{n}+e x_{n-1}+f}, \quad n=0,1, \ldots
$$

with nonnegative parameters and initial conditions such that $A+B+C>0$, and $a x_{n}^{2}+$ $b x_{n} x_{n-1}+c x_{n-1}^{2}+d x_{n}+e x_{n-1}+f>0, n=0,1, \ldots$, the obtained results take the following form.

Corollary 3 Assume that Eq. (25) has the unique positive equilibrium $\bar{x}$. If the following condition holds:

$$
\frac{(|A-a \bar{x}|+|B-b \bar{x}|+|C-c \bar{x}|)(U+\bar{x})+|D-d \bar{x}|+|E-e \bar{x}|}{(a+b+c) L^{2}+(d+e) L+f}<1,
$$

where $L$ and $U$ are lower and upper bounds of all solutions of $E q$. (25) and $f+L>0$, then $\bar{x}$ is globally asymptotically stable on the interval $[0, \infty)$.

In the special case of second order equation with quadratic terms only, we obtain the following result.

Corollary 4 Consider the following equation:

$$
x_{n+1}=\frac{A x_{n}^{2}+B x_{n} x_{n-1}+C x_{n-1}^{2}}{a x_{n}^{2}+b x_{n} x_{n-1}+c x_{n-1}^{2}}, \quad n=0,1, \ldots
$$

with all positive parameters and nonnegative initial conditions such that $a x_{n}^{2}+b x_{n} x_{n-1}+$ $c x_{n-1}^{2}>0$ for all $n \geq 0$. If the following condition holds:

$$
\frac{(|A-a \bar{x}|+|B-b \bar{x}|+|C-c \bar{x}|)(U+\bar{x})}{(a+b+c) L^{2}}<1,
$$

where

$$
\bar{x}=\frac{A+B+C}{a+b+c}, \quad L=\frac{\min \{A, B, C\}}{\max \{a, b, c\}}, \quad U=\frac{\max \{A, B, C\}}{\min \{a, b, c\}},
$$

then the unique equilibrium $\bar{x}$ of Eq. (27) is globally asymptotically stable on the interval $[0, \infty)$

Remark 2 If the strict inequality in conditions (18), (20), (21), (22), and (24) is replaced by equality, then the conclusions of Theorems 4, 5, 6, and Corollary 2 should be changed from global asymptotic stability to stability. 


\section{Competing interests}

The authors declare that they have no competing interests.

\section{Authors' contributions}

All authors contributed equally to the writing of this paper. All authors read and approved the final manuscript.

Received: 10 January 2015 Accepted: 29 May 2015 Published online: 12 June 2015

\section{References}

1. Kulenović, MRS, Ladas, G: Dynamics of Second Order Rational Difference Equations, with Open Problems and Conjectures. Chapman \& Hall/CRC Press, London (2001)

2. Camouzis, E, Ladas, G: Dynamics of Third Order Rational Difference Equations, with Open Problems and Conjectures. Chapman \& Hall/CRC Press, London (2007)

3. Burgić, D, Kalabušić, S, Kulenović, MRS: Period-two trichotomies of a difference equation of order higher than two. Sarajevo J. Math. 4(16), 73-90 (2008)

4. Camouzis, E, Ladas, G: When does local asymptotic stability imply global attractivity in rational equations? J. Differ. Equ. Appl. 12, 863-885 (2006)

5. Palladino, FJ: On the characterization of rational difference equations. J. Differ. Equ. Appl. 15(3), $253-260$ (2009)

6. Kalabušić, S, Kulenović, MRS, Overdeep, CB: Dynamics of the recursive sequence $x_{n+1}=\frac{\beta x_{n-1}+\delta x_{n-k}}{B x_{n-1}+D x_{n-k}}$. J. Differ. Equ. Appl. 10, 915-928 (2004)

7. Bedford, E, Kim, K: Periodicities in linear fractional recurrences: degree growth of birational surface maps. Mich. Math. J. 54, 647-671 (2006)

8. Bedford, E, Kim, K: Dynamics of rational surface automorphisms: linear fractional recurrences. J. Geom. Anal. 19(3), 553-583 (2009)

9. Elabbasy, EM, El-Metwally, H, Elsayed, EM: Global attractivity and periodic character of a fractional difference equation of order three. Yokohama Math. J. 53(2), 89-100 (2007)

10. Elabbasy, EM, El-Metwally, H, Elsayed, EM: On the difference equation $x_{n+1}=\frac{a_{0} x_{n}+a_{1} x_{n-1}+\cdots+a_{k} x_{n-k}}{b_{0} x_{n}+b_{1} x_{n-1}+\cdots+b_{k} x_{n-k}}$. Math. Bohem. 133, 133-147 (2008)

11. Kocic, VL, Ladas, G: Global Behavior of Nonlinear Difference Equations of Higher Order with Applications. Kluwer Academic, Dordrecht (1993)

12. Kulenović, MRS, Merino, O: Discrete Dynamical Systems and Difference Equations with Mathematica. Chapman \& Hall/CRC Press, London (2002)

13. Kulenović, MRS, Merino, O: A global attractivity result for maps with invariant boxes. Discrete Contin. Dyn. Syst., Ser. B 6, 97-110 (2006)

14. Sedaghat, H: Nonlinear Difference Equations. Theory with Applications to Social Science Models. Mathematical Modelling: Theory and Applications, vol. 15. Kluwer Academic, Dordrecht (2003)

15. Jašarević, S, Kulenović, MRS: Basins of attraction of equilibrium and boundary points of second order difference equation. J. Differ. Equ. Appl. 20, 947-959 (2014)

16. Kalabušić, S, Kulenović, MRS, Mehuljić, M: Global period-doubling bifurcation of quadratic fractional second order difference equation. Discrete Dyn. Nat. Soc. 2014, Article ID 920410 (2014)

17. Kulenović, MRS, Pilav, E, Silić, E: Local dynamics and global attractivity of a certain second order quadratic fractiona difference equation. Adv. Differ. Equ. 2014, Article ID 68 (2014)

18. Dehghan, M, Kent, CM, Mazrooei-Sebdani, R, Ortiz, NL, Sedaghat, H: Dynamics of rational difference equations containing quadratic terms. J. Differ. Equ. Appl. 14, 191-208 (2008)

19. Dehghan, M, Kent, CM, Mazrooei-Sebdani, R, Ortiz, NL, Sedaghat, H: Monotone and oscillatory solutions of a rational difference equation containing quadratic terms. J. Differ. Equ. Appl. 14, 1045-1058 (2008)

20. Kent, $\mathrm{CM}$, Sedaghat, $\mathrm{H}$ : Global attractivity in a quadratic-linear rational difference equation with delay. J. Differ. Equ. Appl. 15, 913-925 (2009)

21. Kent, $\mathrm{CM}$, Sedaghat, $\mathrm{H}$ : Global attractivity in a rational delay difference equation with quadratic terms. J. Differ. Equ. Appl. 17, 457-466 (2011)

22. Sedaghat, $\mathrm{H}$ : Global behaviours of rational difference equations of orders two and three with quadratic terms. J. Differ. Equ. Appl. 15, 215-224 (2009)

23. Janowski, EJ, Kulenović, MRS: Attractivity and global stability for linearizable difference equations. Comput. Math. Appl. 57(9), 1592-1607 (2009)

24. Camouzis, E, Ladas, G, Palladino, F, Quinn, E: On the boundedness character of rational equations. Part 1. J. Differ. Equ. Appl. 12, 503-523 (2006)

25. Brett, A, Janowski, EJ, Kulenović, MRS: Global asymptotic stability for linear fractional difference equation. J. Differ. Equ. 2014, Article ID 275312 (2014) 